\section{LOS LÍMITES AL CRECIMIENTO, EL CAMBIO CLIMÁTICO Y LA INNOVACIÓN}

\author{
M. ${ }^{a}$ Carmen Gallastegui Zulaica \\ Departamento de Análisis Económico I \\ Instituto de Economía Pública. UPV/EHU \\ mariacarmen.gallastegui@ehu.es
}

\begin{abstract}
The purpose of this article is to analyze what the environment and its' management represents in terms of social innovation; the restrictions that the environment and planet care imply, the actions that have been adopted, the ways in which the different companies have reacted and what the future still holds. We will present this analysis by using to main examples: on the first hand, the problem concerning the limits to growth; on the other hand, the problem of climate changes. The solutions that are being proposed, from the mitigation, the use of market incentives (prices and taxes), the creation of markets for the rights to pollution, the "geo-engineer" solutions, the impulse for technologic innovation, are all clear examples of the ways in which society is confronting the problem.
\end{abstract}

KEY WORDS: Environment; social innovation; climate change; limits; growth.

\section{INTRODUCCIÓN}

Los economistas comenzamos a preocuparnos por la cuestión de la escasez de los recursos naturales hace ya mucho tiempo. Jevons ${ }^{1}$ analizó la posibilidad de que hubiera una escasez de carbón en Inglaterra. Malthus ${ }^{2}$ se preocupó del aumento poblacional y la escasez de alimentos y no son los únicos autores relevantes en esta área del pensamiento.

El análisis, más general del uso de los activos ambientales ha sido, sin embargo, más tardío. Durante los sesenta, y gracias básicamente al trabajo pionero de $\mathrm{A}$. Pigou ${ }^{3}$, Baumol y Oates ${ }^{4}$ llegamos a la conclusión de que disponiamos de una buena estructura a través de la cual modelar la contaminación originada por las empresas. Durante los últimos treinta años los economistas hemos aprendido, entre otras cosas, que si bien las empresas son generadoras de efectos externos, no son los únicos agentes que producen problemas medioambientales. Los consumidores, con el uso excesivo de recursos naturales, la eliminación indiscriminada de residuos, la generación de congestión en las carreteras y

\section{LIMITS TO GROWTH, CLIMATE CHANGE AND INNOVATION}

RESUMEN: Este artículo tiene como objetivo analizar lo que el medio ambiente y su gestión representa en términos de innovación social; las restricciones que el cuidado del medio ambiente y del planeta plantean, las medidas que se han adoptado, la forma en que las empresas han reaccionado y lo que nos depara todavia el futuro. Lo haremos utilizando dos ejemplos básicos: por un lado, el problema de los Límites al Crecimiento y por otro, el problema del Cambio Climático. Las soluciones que se están proponiendo, desde la mitigación, el uso de incentivos de mercado (precios e impuestos), creación de mercados de derechos de contaminación, soluciones "geo-ingeniereles", impulso a la innovación tecnológica son claros exponentes de la forma en que la sociedad está enfrentándose al problema.

PALABRAS CLAVE: Medio ambiente; innovación social; cambio climático; límites; crecimiento.

sus efectos sobre la salud, y la emisión de $\mathrm{CO}_{2}$, somos otro foco generador de problemas en el medio ambiente al que necesariamente hay que tener en cuenta.

Sirva esta última reflexión como advertencia de que los problemas ambientales se han convertido en algo tan acuciante $y_{1}$ en muchos casos de naturaleza tan global que el tipo de análisis y las medidas necesarias para abordarlos e intentar solucionarlos son, hoy en día, mucho más sofisticados. Además los esfuerzos analíticos y de modelización que se están llevando a cabo reconocen la complejidad de la tarea y la necesidad de análisis multidisciplinares.

Este artículo tiene como objetivo analizar lo que el medio ambiente y su gestión representa en términos de innovación social; las restricciones que el cuidado del medio ambiente y del planeta plantean, las medidas que se han adoptado, la forma en que las empresas han reaccionado y lo que nos depara todavía el futuro. Lo haremos utilizando dos ejemplos básicos: por un lado, el problema de los Límites al Crecimiento y por otro, el problema del Cambio 
Climático (CC). Ambos están relacionados en tanto que las emisiones de gases de efecto invernadero (GEI) y crecimiento económico tienen una correlación positiva.

\section{El enfoQue teóRICO}

Las actividades económicas tienen lugar, y son parte, del sistema que constituye la tierra y la atmósfera. Este sistema denominado "el medio ambiente natural" tiene a su vez otro medio ambiente: el resto del universo. Las interdependencias entre la economía y el medio ambiente son múltiples. Las empresas obtienen factores productivos del medio ambiente: agua, aire, minerales, recursos pesqueros $y$, una vez que se producen los bienes de consumo y los servicios demandados por el mercado, los humanos depositamos residuos cumpliendo, por tanto, el medio ambiente con otra importante función: la de receptor de estos residuos.

El medio ambiente es un sistema cerrado y las leyes de la termodinámica indican que intercambia energía, pero no materia, con su propio medio ambiente. Esto, que a menudo olvidamos los economistas, constituye una ley básica importante.

Las empresas productoras de bienes de consumo y de bienes de inversión, los consumidores individuales que demandamos todo tipo de bienes y servicios, y el medio ambiente, con sus servicios recreativos, de proveedor de recursos productivos y de receptor de residuos, interaccionan entre sí de formas muy variadas.

Además, muchos de los factores productivos utilizados por las empresas constituyen recursos para los que no existen derechos de propiedad asignados. Como consecuencia la inexistencia de mercados implica que no hay precios que guíen las decisiones acerca de cuánto utilizar y cómo utilizar los bienes ambientales. El corolario inmediato es que, la gratuidad, incentiva la utilización de cantidades excesivas de estos bienes. Se produce una sobreexplotación e incluso un agotamiento de recursos importantes para la vigencia de los ecosistemas y la bio-diversidad.

La teoría de los bienes/males públicos desarrollada por P. Samuelson ${ }^{6}$ en los sesenta constituye un buen anclaje para entender porqué se producen ineficiencias si no hay regulación a la hora de decidir qué pueden y no pueden utilizar las empresas y los consumidores de los bienes y servicios ambientales. Para Samuelson, los bienes públicos a diferencia de los bienes privados, son aquéllos que presentan dos características básicas, la no rivalidad en el consumo y la imposibilidad de exclusión. El clima de la tierra es un bien no rival en el consumo y nadie tiene posibilidad de excluirse del mismo. P. Samuelson probó, además, que en presencia de bienes públicos se produce una infraprovisión de los mismos por parte de los mercados. En el caso del CC esto se concreta en el hecho de que no se puede confiar en que el mecanismo de mercado provea un clima de la tierra estable.

Otra forma de visualizar el problema del medio ambiente es la seguida por A. Pigou quien modeló la contaminación como un efecto externo negativo. En su concepción las empresas, a la hora de tomar sus decisiones de producción y de utilización de factores productivos, maximizan sus beneficios privados. Al no tener en cuenta el efecto de sus acciones sobre el resto del sistema, generan efectos externos (polución y eliminación de residuos) sin que los costes que estos efectos generan estén incluidos en sus procesos de decisión. La solución propuesta por Pigou cae de su peso. Se trata de que estos efectos externos sean "internalizados", de forma tal que se generen los incentivos correctos para que los agentes económicos tomen en cuenta todos los costes, tanto internos como externos, que sus decisiones ocasionan.

Inicialmente el análisis de este tipo de fenómenos se realizaba en contextos de equilibrio parcial y teniendo en cuenta sólo los flujos de las emisiones. Además el contexto utilizado era estático. Los desarrollos en diversas direcciones no se hicieron esperar.

El trabajo pionero de W. Baumol y Oates ${ }^{7}$ sentó las bases para poder llevar a cabo el análisis teórico en un marco 
de equilibrio general donde las empresas generan efectos externos (polución) de naturaleza pública (se puede sumar la contaminación que realizan todas y cada una de las empresas sin importar la fuente de donde proceden las emisiones) y donde los consumidores y las propias empresas sufren las consecuencias que la suma de todos los residuos emitidos generan sobre sus funciones de producción y de utilidad.

Cuando en lugar de tener en cuenta sólo las emisiones flujo se tienen también en cuenta los efectos stock (muchos de los contaminantes perviven en el medio en que se depositan y se acumulan a lo largo del tiempo convirtiéndose en un stock de polucionantes), el análisis es algo más complejo, en tanto que requiere de una aproximación dinámica, pero las enseñanzas básicas que aprendimos con Pigou, Baumol y Oates, aunque precisan de matizaciones, siguen siendo válidas.

Similarmente cuando la contaminación ocasionada por las empresas no es de naturaleza pública, lo que implica la "fuente emisora" importa y nos enfrentamos a un vector de variables que representan la contaminación de cada empresa, las conclusiones varian y en lugar de un único impuesto Pigouviano es necesario disponer de un vector de impuestos, dependiendo del daño que las emisiones de cada fuente ocasionan. La solución de internalización de los costes externos sigue utilizando "precios" (impuestos) pero, en lugar de un único impuesto son necesarios varios tipos diferentes.

Sirvan estas ideas como ilustración de que en el área de la economía ambiental la caracterización de la polución como efecto externo y en ocasiones como "mal público" produjo innegables frutos.

\subsection{La aproximación de estándares y el teorema de coste mínimo}

Una vez constatado que conseguir resultados "eficientes" en el sentido de W. Pareto ${ }^{8}$, en un contexto en que existían efectos externos era muy difícil por no decir imposible, la economía se planteó la necesidad de formular objetivos medioambientales que lograran una eficacia ambiental sin necesidad de llegar a la eficiencia.

La respuesta fue inmediata. Si conseguir eficiencia es muy costoso en términos de información, al ser necesario dis- poner de evaluaciones de todos los costes y beneficios lo que, a su vez, obliga a conocer las funciones de utilidad y las funciones de coste de todas las empresas y consumidores, deberiamos buscar otro objetivo ambiental y no necesariamente el logro de la eficiencia. El cambio de paradigma de la eficiencia a la eficacia es sustancial. Como ejemplo supongamos que un ayuntamiento conoce que, a partir de un cierto nivel de emisiones, la contaminación generada por una empresa produce daños perversos en la salud de sus vecinos. Decide, con el consejo experto de los que conocen la relación entre salud y emisiones, cual es el nivel de emisiones que evitaría los daños sobre la salud y decide poner como objetivo que este límite de emisiones no se supere.

Se trata, en estas circunstancias, de averiguar, qué instrumentos son capaces de conseguir los estándares ambientales incurriendo en un coste mínimo; o, en otras palabras, se trata de investigar cuales son los instrumentos que satisfacen la propiedad de ser "coste efectivos".

Los resultados no se hicieron esperar demostrándose que los instrumentos de mercado, cánones, impuestos y algunos más novedosos cumplían con esta propiedad.

Se probó, también, que sí se quería recurrir a los instrumentos más modernos de "permisos" de contaminación y de creación de mercados para estos permisos, la posibilidad de que éstos pudieran ser negociables o intercambiables entre empresas, permitiría que los resultados fuesen satisfactorios desde el punto de vista económico.

Hoy existe un consenso a la hora de reconocer la superioridad, en términos de efectividad en costes, de los instrumentos de mercado. Se trata de admitir, primero, que las empresas no tienen porqué ser idénticas en cuanto a los costes que incurren a la hora de reducir sus niveles de emisión, y segundo, de conseguir que, aceptando estas diferencias, se logre un sistema que permita reducir las emisiones globales de la forma menos costosa para la sociedad. Para ello interesa que las empresas, para las que resulten más barato reducir sus emisiones lo hagan en mayor cuantía que aquéllas para las cuales la reducción es más costosa.

Al mismo tiempo que estos resultados se trasladaban a los responsables políticos las empresas, conscientes de 
que principios como el que "contamina paga" o el "principio de precaución" aparecían en la escena económica y ambiental comenzaran a tomar en cuenta el hecho de que debían cambiar su comportamiento con respecto al medio ambiente. Aparecieron propuestas que se materializaron en lo que se ha denominado en la literatura los "acuerdos voluntarios"; el Gobierno fija los objetivos ambientales, pero permite que sean las propias empresas las que decidan cómo se adaptan para poder cumplir con los mismos. Aparecen también otras alternativas. Por citar sólo algunas, la aproximación que implica utilizar la información de forma tal que sean las propias empresas, las que deseosas de mantener una "reputación" ambiental adecuada decidan controlar sus emisiones. Tener una credibilidad de agente "verde" o "ambientalmente responsable" puede aportar beneficios netos.

La investigación en este campo, avanzó de forma rápida. No basta con conseguir que los resultados sean eficaces en un contexto estático, sino que es preciso lograr el difícil objetivo de la "eficiencia dinámica". Este objetivo aparece ligado al hecho de la renovación tecnológica y al uso de las tecnologías limpias. La elección de los instrumentos de acción debe tener en cuenta que lo que interesa es no sólo que las empresas reduzcan, de forma eficaz y minimizando costes, sus emisiones actuales sino que, además, se vean incentivadas a efectuar cambios en sus procesos productivos de forma tal que sus emisiones futuras se vean reducidas. Los instrumentos ambientales deben ser juzgados atendiendo a este principio de la eficiencia dinámica y no todos los utilizados en la práctica lo consiguen. El mismo principio se puede aplicar a los consumidores.

Esta descripción estilizada de lo que se ha avanzado en economía ambiental y en el terreno de la política ambiental no pretende hacer justicia a toda la literatura existente. Pero hay una última cuestión de interés.

Las políticas ambientales han sido vistas, durante demasiado tiempo, como una limitación para el logro del crecimiento de las empresas, la generación de empleo y la obtención de beneficios. Esta visión resulta, a mi entender, demasiado negativa y pesimista. La otra cara de la moneda, la posibilidad de que la protección ambiental genere nuevas oportunidades de negocio, permita la búsqueda de nuevos nichos de mercado y sea incluso generador de empleo a nivel global es una visión que no puede ser descartada.

Se han adelantado argumentos en el sentido de que reglamentaciones ambientales excesivamente severas, que hacen aumentar los costes de producción de las empresas podian generar movimientos de las empresas de unas zonas a otras. Sin duda esta consideración es relevante, pero quizá más en teoría que en la práctica. A fin de cuentas, parece probado que los requisitos ambientales sólo en ocasiones muy especiales presentan las características de ser determinantes a la hora de elegir una determinada localización.

Por otro lado, la política ambiental, en sus aspectos más centrales, está dictada hoy desde la UE por lo que las implicaciones, en términos de localización de empresas, dentro de la zona geográfica de la UE ha dejado de ser relevante. Hay un argumento, eso sí, que puede afectar a zonas del planeta menos desarrolladas y es preciso tener en cuenta; el denominado efecto del "garbage out of my garden". Cuando el tipo de efecto ambiental reúne las características de "bien privado" -hay rivalidad en el consumo y posibilidad de exclusión-, los análisis teóricos son distintos que cuando las emisiones tienen las caracteristicas de bien/mal público. El ejemplo más claro lo constituye la "Iluvia ácida" causada por emisiones de $\mathrm{SO}_{2}$ o la eliminación de residuos de naturaleza radioactiva. En el primer caso es evidente que el efecto externo causado es de naturaleza rival. Si la lluvia ácida cae en los bosques de la Selva Negra no perjudica a los bosques de otras zonas limitrofes. El tipo de solución que hay que buscar ante esta situación es distinta a la resultante cuando las emisiones tienen la naturaleza de bien público. El ejemplo de los residuos radiactivos es también ilustrativo. Se trata de un efecto externo de naturaleza "desplazable". Los residuos radiactivos que han viajado para ser enterrados en los cementerios construidos a tal fin o los que viajan a paises menos desarrollados que están dispuestos a admitirlos si se les paga por ello, constituyen un ejemplo. Si nadie quiere la basura dentro de sus márgenes territoriales y si, para depositarlo en algún lugar, es preciso pagar por ello aparece, además de la variable espacio, la variable nivel de renta y con ella los problemas redistributivos a los que todavía no hemos hecho referencia, pero que son, sin lugar a dudas, importantes. 
La eficacia ambiental, la eficiencia económica y la equidad o los aspectos distributivos no siempre funcionan en la misma dirección. Por ello, a menudo no basta con una única política sino que es preciso diseñar paquetes de medidas para poder "compensar" los efectos distributivos que las diversas políticas imponen. También es verdad, sin embargo, que las restricciones que la consecución de un medio ambiente saludable exige, generan oportunidades de negocio, incentivos a cambiar de tecnologias que pueden conseguir que las empresas no sólo sean ambientalmente más "amistosas" sino también más eficaces desde una perspectiva exclusivamente económica. Tampoco puede olvidarse las actividades de investigación y desarrollo que el cuidado del medio ambiente está impulsando. Este tipo de argumentos aparecen recogidos en Gallastegui y Escapa9.

\section{Los límites al Crecimiento}

El primer Informe Meadows acerca de los Límites al Crecimiento publicado en 1972 a instancias del Club de Roma constituyó un aldabonazo que abordó las implicaciones del crecimiento poblacional y del crecimiento económico para los servicios y activos proporcionados por el planeta Tierra.

El Informe, criticado por muchos, y alabado por otros, fue desde el punto de vista económico, objeto de controversias en tanto que no incluía en su formulación variables económicas como los precios de los factores naturales, ni las posibilidades de sustitución entre el capital creado por el hombre y el capital natural. La confianza de los economistas en estos procesos de sustitución así como en los avances tecnológicos que los incrementos en precios de las materias primas iban a precipitar, debilitó, por decirlo de forma suave, las predicciones más negativas del Informe.

Treinta años después, Meadows et al. ${ }^{10}$, retomaron el análisis de las interacciones entre crecimiento de la población, crecimiento económico y los límites que los activos naturales del planeta imponen, aceptando muchas de las críticas que su Informe preliminar había suscitado.

Sus posiciones, más matizadas y obtenidas a través de la utilización de un modelo de dinámica de sistemas de- nominado World 3, tuvieron, quizá, un menor impacto, pero paradójicamente fueron también sino más creíbles sí, al menos, consideradas como menos alarmistas para un subconjunto de la población y de los políticos preocupados por el logro de sendas de crecimiento sostenibles y por las extralimitaciones que el comportamiento humano origina en el medio natural.

Los años transcurridos entre el primer y el último Informe han sido cruciales para el análisis de los problemas ambientales y de los recursos naturales, años en los que se han puesto sobre el tapete problemas tan globales y vitales como el del agujero de la Capa de Ozono, la escasez de recursos hídricos en ciertas zonas del planeta, el Calentamiento Global, la desertización y la erosión o la pérdida de biodiversidad, por citar algunos de los más importantes.

El último Informe, titulado "Los Límites del Crecimiento 20 años después" aborda la misma cuestión que los autores se plantearon en el estudio del año 1972, utilizando un modelo estadístico más completo y con interrelaciones más sofisticadas y llegando a conclusiones muy preocupantes.

Su ámbito de aplicación es el planeta Tierra y los datos que utilizan se refieren, por tanto, al contexto más macro y agregado posible: el mundo en su conjunto.

\section{LAS EXTRALIMITACIONES}

El rápido crecimiento demográfico y económico, la aceleración en el crecimiento, el cambio rápido, junto con la existencia de barreras o límites que impiden el correcto funcionamiento de los sistemas naturales dinámicos y la posibilidad de errores de percepción en las respuestas encaminadas a mantener el sistema dentro de sus límites, son las tres condiciones necesarias y suficientes para que se produzca una extralimitación.

No siempre las extralimitaciones son lesivas sino que constituyen, en numerosas ocasiones, un fenómeno común que adopta numerosas formas. Ocasionalmente surge el potencial de una extralimitación catastrófica que confronta a la humanidad con consecuencias potenciales sumamente 
peligrosas que, además, exigen mucho tiempo para que puedan ser subsanadas.

Las proyecciones demográficas, las proyecciones económicas y la capacidad de carga de nuestro hábitat, constituyen los tres elementos básicos que han de ser tenidos en cuenta en una perspectiva sistémica que permita acercarse a conocer los Límites al Crecimiento. Para sostener el crecimiento económico y demográfico del mundo o de un área geográfica concreta la lista de inputs necesaria es larga y diversa. En el informe Meadows esta lista aparece clasificada por categorias:

Categoría 1: Se incluyen todas las necesidades físicas que sostienen la actividad biológica e industrial. En esta categoria deben incluirse factores como:

- tierras fértiles,

- minerales

- metales,

- energía,

- clima moderado,

- sistemas ecológicos capaces de absorber residuos.

Categoría 2: Se incluyen las necesidades sociales:

- paz y estabilidad social,

- justicia y seguridad personal,

- dirigentes honestos y de amplias miras,

- educación y mentalidad abierta a nuevas ideas,

- disposición a admitir errores,

- disposición a experimentar,

- bases institucionales adecuadas.

Una forma que sirve para funcionar con estas categorias y ayuda, al mismo tiempo, a definir los límites sostenibles del caudal productivo, del caudal de energía y de los materiales, es la propuesta por H. Daly"1". Se resume en tres reglas que han de cumplirse si no se desea llegar a "extralimitaciones" que puedan originar resultados catastróficos.

Las reglas son las siguientes:

\section{Primera}

Para todos los recursos renovables (los que están sujetos a sus propias leyes de reproducción y crecimiento) es preciso que las extracciones o capturas no sean nunca superiores a las tasas de crecimiento naturales de los recursos considerados. No se puede capturar, cazar o talar más que lo que los recursos subyacentes crezcan de forma intrinseca y natural. Es el concepto de sostenibilidad más antiguo y menos ambiguo.

\section{Segunda}

Para todos los recursos no renovables (combustibles fósiles, minerales, aguas freáticas, fósiles...) la tasa de consumo sostenible es siempre igual o inferior a la tasa a la que un recurso renovable lo pueda sustituir ${ }^{12}$. Esta regla no es tan intuitiva como la primera y ha de enfrentarse al hecho de que, en el límite, los recursos no renovables realmente no existen. Los avances tecnológicos y los costes y precios siempre harán posible que puedan ser sustituidos por otros 0 que llegar al agotamiento sea difícil aunque no imposible.

\section{Tercera}

Para los contaminantes, la tasa de emisión sostenible ha de ser menor o igual a la tasa a la que el contaminante puede ser reciclado, absorbido o neutralizado en su sumidero.

Comprobar que la gestión le exige garantizar que las tres reglas de Daly se cumplan fuente por fuente, sumidero por sumidero y recurso por recurso.

La forma en que este tipo de análisis se ha ido concretando en la literatura es a través de los cálculos de la carga humana sobre el Medio Ambiente también denominada "Huella Ecológica" (M. Wackernagel) ${ }^{13}$ que evalúa "el impacto total de la humanidad en la naturaleza". La Huella ecológica debe tener en cuenta todos los efectos, es decir debe incorporar en su definición desde la extracción de recursos a la destrucción de la biodiversidad pasando por la emisión de contaminantes, el consumo de energía o la urbanización de los terrenos vírgenes así como la destrucción de la biodiversidad. Esto implicaría que todas las demandas humanas sobre el ecosistema se convertirian en un número equivalente de hectáreas necesarias para sostener el servicio ecológico prestado por un tiempo indefinido.

El deterioro ecológico podría medirse utilizando la conocida expresión IPAT que se concreta en la fórmula:

$$
I=\text { P.A.T. }
$$


Donde,

I = huella ecológica 0 impacto

$\mathrm{P}=$ Población

$A=$ Nivel de abundancia

$\mathrm{T}=$ Daño causado por las tecnologías empleadas para sostener esa abundancia

Afortunadamente el margen de mejora existente para reducir este deterioro ecológico es asombroso o, en otros términos, el impacto humano en las fuentes y sumideros del planeta podrían reducirse en un grado elevado.

Sin embargo, no es tan fácil elegir entre las distintas posibilidades a no ser que el análisis que se lleve a cabo pase de ser un análisis estático a contemplar la vertiente dinámica que los problemas de explotación de los recursos naturales plantean. En lugar de un análisis de factor a factor es preciso llevar a cabo un análisis del conjunto del sistema. Y esto es precisamente lo que Meadows et al., abordaron en su último trabajo. El modelo World3 utilizado es complejo y hace un seguimiento de magnitudes como la población, el capital industrial, la contaminación persistente y la superficie de tierra cultivada. En el modelo, las magnitudes varían a través de variables flujos (tasa de natalidad y defunción, inversiones y amortizaciones, emisión y asimilación de contaminación, erosión del suelo, calificación de terrenos para usos urbanos e industriales, etc.).

Se contemplan, además, interrelaciones entre las distintas variables y entre las diferentes relaciones. Así, un cambio poblacional puede originar un cambio en la economía, lo que a su vez afectará a las tasas de mortalidad y de natalidad. El modelo incluye, por tanto, ciclos de realimentación admitiendo que no todas las relaciones tienen que ser lineales (por poner un ejemplo: si los consumidores eligen primero los productos de menor coste y las empresas minimizan los costes de producción, las últimas tierras que se dediquen al cultivo serán las menos fértiles y el coste de los alimentos aumentará de forma no lineal).

El modelo incluye también la posibilidad de desfases y retardos.

La pregunta central que World3 pretende contestar es: ¿Cómo van a interactuar la población mundial y la eco- nomía material en expansión con la capacidad de carga limitada de la Tierra y cómo se adaptaran a ella en los próximos decenios?

Según los autores de "Los Límites del Crecimiento" hay pruebas evidentes de que la sociedad mundial ha superado ya su capacidad de carga por lo que la pregunta relevante sería, ¿qué política incrementará las posibilidades de una transición suave de retorno a los límites planetarios?

Las preguntas que pretende contestar World3 tienen su analogía en las que ahora constituyen una preocupación para muchos científicos $y$, en particular, para los que trabajan en cuestiones de Cambio Climático.

En términos del Cambio Climático (CC), paradigma de alteración en el clima de la tierra generado por las emisiones humanas de gases de efecto invernadero (GEI), nos preguntaríamos: ¿qué políticas o que combinación de políticas hemos de poner en práctica para conseguir que las emisiones no superen los 450ppm de forma tal que podamos garantizar (con la inherente incertidumbre en estas materias) que la temperatura media del Planeta no experimente un ascenso superior a los $2^{\circ}$ centígrados? Esta cuestión se aborda en la próxima sección.

\section{La economía del Cambio Climático}

La teoría económica aplicada al análisis del CC es clara y contundente. El cambio en el clima de la tierra constituye un fracaso de mercado, bien porque se considera que las emisiones de $\mathrm{GEl}$ y en particular de $\mathrm{CO}_{2}$ generan efectos externos perniciosos que tiene efectos de naturaleza global, bien porque el Clima de la Tierra se modela como un bien público y la estabilidad del clima está en peligro porque los bienes públicos de naturaleza global constituyen, tal y como antes se ha mencionado, ejemplos típicos donde se producen fracasos de mercado ${ }^{14}$. Las soluciones a este fracaso no son sencillas.

Se ha optado, al menos en el caso en que Europa se ha involucrado de forma más activa, por el logro de acuerdos "vinculantes" o de mecanismos que obliguen a los paises a adoptar decisiones vinculantes en torno al recorte de las emisiones. 
En la práctica esto ha significado la definición y aprobación de Protocolos (Kyoto), la existencia de una Cooperación parcial, el desarrollo de Mecanismos de Acción Limpia, y la constitución de nuevos mercados donde se intercambian derechos a contaminar basados en el sistema de "cap" and "trade".

Pero todos estos desarrollos no son suficientes. Sería necesario, además, políticas que diseñaran y crearan Consejos Público-Privados, la incorporación del Clima de la Tierra y el efecto de las emisiones en las decisiones de las empresas, de los organismos públicos y en la sociedad civil. Y, obviamente, será también preciso desarrollar la innovación tecnológica que permita descubrir alternativas a través de las que se pueda proteger el clima, a través de métodos como los de Captura y Secuestro de Carbono o las propuestas de fertilización de los océanos y otras propuestas englobadas bajo el nombre de "Soluciones Geo-ingenieriles" sobre las que se está investigando mucho en la actualidad.

La política educativa y formativa con los cambios en los $\mathrm{CV}$ académicos, el aprendizaje de una gestión eficiente, en términos ambientales, en los centros escolares, universitarios (experiencias en este sentido no faltan) constituyen también alternativas imprescindibles.

El mayor contacto con la naturaleza de las generaciones más jóvenes y una reflexión serena en torno a la cuestión del crecimiento económico, su correlación con las emisiones, los niveles de calidad de vida y de PIB per cápita son también imprescindibles.

\subsection{La importancia de la energía}

Detrás del CC se encuentra el cambio del paradigma energético. Esta idea, ampliamente aceptada. Pero no podrá hacerse sin cambiar el contexto de las políticas económicas y ambientales existentes. Los aspectos decisivos, a mi juicio, no son la tecnología o la escasez de recursos, sino las instituciones, reglas, mecanismos de financiación así como las regulaciones necesarias para conseguir que los mercados operen en apoyo de la energía necesaria para garantizar un desarrollo sostenible.

Avanzar hacia combustibles con menos carbono va contra la abundancia relativa de recursos fósiles: carbón, el más abundante, seguido por el petróleo y el gas natural. Descarbonizar implica deshacerse del carbono en exceso.
La figura 1 aborda esta terminología tratando de abarcar desde el sistema energético a la cadena energética, pasando por la energía primaria.

FIGURA 1. UN EJEMPLO DE LA CADENA ENERGÉTICA DESDE LA EXTRACCIÓN A LOS SERVICIOS

\begin{tabular}{|ccc|}
\hline $\begin{array}{c}\text { Sistema energético } \\
\text { Extracción y tratamiento }\end{array}$ & $\rightarrow$ & $\begin{array}{c}\text { Sector energético } \\
\text { Mina de carbón }\end{array}$ \\
Energía primaría & $\rightarrow$ & Carbón \\
Tecnologías de conversión & $\rightarrow$ & Central termoeléctrica \\
Tecnologías de distribución & $\rightarrow$ & Planta de cogeneración \\
Energía final & $\rightarrow$ & Red \\
Tecnología de uso final 1 & $\rightarrow$ & Sistema de arco eléctrico \\
Energía útil & & Calor de fusión \\
Tecnologías de uso final 2 & $\rightarrow$ & Horno \\
Servicios energéticos & & Producción de acero \\
\end{tabular}

Fuente: Informe Mundial de la Energía. Programa de las Naciones Unidas para el Desarrollo. Madrid, abril 2001.

Durante los últimos 30 años, el PIB mundial creció en una tasa próxima al $3 \%$ anual en promedio, mientras que las emisiones de carbono crecieron la mitad de rápido. Así, el cociente entre emisiones y dólares de PIB (la intensidad de carbono) cayó en torno a 1,5\% al año. Para que las emisiones globales sean las mismas dentro de 50 años, la intensidad de carbono deberá disminuir igual de rápido que el crecimiento de la economía.

Por supuesto dicha tarea requerirá nueva ciencia y nueva ingenieria para recortar los costes y afrontar los problemas que acompañan inevitablemente el empleo de nuevas tecnologías. Pero mantener las emisiones dentro de cincuenta años en su nivel actual, sin estrangular el crecimiento es un resultado deseable y díficil. Por eso la pregunta de ¿qué conjunto de políticas proporcionarán el resultado perseguido? sigue siendo relevante.

Es imprescindible disponer de instituciones que transmitan de manera fiable un precio de las emisiones de carbono presentes y futuras. A fin de cuentas ninguna tecnología podrá nunca competir contra la "alternativa" de liberar gratis el $\mathrm{CO}_{2}$ en la atmósfera. $Y$ esto, por sí mismo, puede no ser suficiente. Es posible que los Gobiernos precisen además es- 
timular la comercialización de tecnologías bajas en carbono para elevar el número de opciones competitivas disponibles en el futuro (eólica, fotovoltaica, coches hibridos...).

Éste es un tema que junto con otros aspectos está tratado en (Gallastegui y Chamorro) ${ }^{15}$.

\section{LAS POLÍticas Y LOS AgENTES IMPLICADOS}

La respuesta a la pregunta planteada al final de la sección III y otras muy similares requiere tener en cuenta numerosos aspectos lo que hace que tanto el problema de los Límites al Crecimiento como el del Cambio Climático hayan de abordarse de forma multidisciplinar. Se trata de utilizar modelos globales para predecir resultados globales a nivel del Planeta Tierra. Dentro de estos modelos globales existen distintas alternativas cuando se desea incorporar aspectos económicos, energéticos, ambientales, demográficos... La diferenciación puede establecerse en términos de qué aspectos son incorporados y cuáles dejados fuera del análisis, pero también en términos de la metodología utilizada en su elaboración. No entraré en este análisis por problemas de espacio.

En el caso de estudio del Cambio Climático, por ejemplo, modelar el sector energético en detalle es crucial. La innovación que este proceso ha hecho surgir en diferentes sectores ha sido inmensa. Pero es que, además, la concienciación social que se ha producido y las decisiones adoptadas por muchos ciudadanos responsabilizados con el tema permite decir que la innovación ha tenido éxito gracias a la participación que la demanda selectiva de los consumidores informados ha tenido en el proceso. Sin olvidar, claro está, los procesos de incentivos que el sector público ha puesto en marcha, como los planes RENOVE.

\section{Conclusiones}

Este artículo ha pretendido ilustrar cómo el conocimiento de los problemas ambientales y la necesidad de impul- sar acciones para resolverlos han servido para desarrollar mecanismos de actuación que, en sí mismos, constituyen innovaciones que benefician a la sociedad. De no saber qué hacer ante la pérdida de recursos que imponía límites al crecimiento del PIB o cómo abordar el problema del cambio climático hemos pasado a situaciones en las que en Europa existen mecanismos innovadores que permiten que los agentes económicos intercambien sus derechos de emisión. Los mercados de cap and trade. "Cap" porque se establece un límite a las emisiones y "trade" porque se permite que los permisos, que se distribuyen como derecho a emitir y que no pueden sobrepasar en total al "cap" decidido, puedan ser intercambiados en un mercado están funcionando. Se han desarrollado, además, nuevas instituciones y reglas. Por citar las más importantes, el establecimiento de derechos de propiedad sobre áreas medioambientes de propiedad común, la zonas económicas exclusivas (ZEE) en los océanos o la creación de mecanismos de valoración para bienes en principio intangibles.

Y hay más. Las eco-etiquetas, las organizaciones ecologistas que luchan por preservar valores como la biodiversidad, el clima de la tierra, la calidad de las aguas de los océanos, etc., sin olvidar los progresos tecnológicos y las soluciones geo-ingenieriles que la necesidad de preservar el clima de la tierra están propiciando. La captura y secuestro de carbono, la fertilización de los océanos, la construcción de cementerios de residuos, el reciclaje...; todas estas acciones, inconcebibles hace menos de 50 años, han sido posibles gracias a que la sociedad se ha dado cuenta de que preservar los flujos y beneficios que el planeta tierra nos proporciona es tan importante para nuestro bienestar como preservar el flujo monetario en el sistema económico para conseguir mantener el crecimiento y el progreso económico. Y no olvidemos todo lo que se ha ido construyendo en torno al concepto de sostenibilidad, concepto que en su acepción original surgió como respuesta al agotamiento de los recursos naturales y al peligro evidente de que las generaciones futuras no iban a poder disfrutar de los mismos recursos que los que hemos disfrutado las generaciones precedentes y las presentes. 
1 Jevons, W. S. (1865): The coal question: An inquiry Concerning the Progress of the Nation and the Probable Exhaustion of our Coal Mines, Flux, W. (ed).

2 Malthus, T. R. (1798): Ensay sobre el principio de la población.

3 Pigou, A. (1920): The Economics of Welfare, London: MacMillan.

4 Baumol, W. J. y Oates, W. E. (1988): The Theory of Environmental Policy, Externalities, Public Outlay and the Quality of Life, Prentice Hall, Englewood Cliffs, NJ.

5 Meadows, D. H.; Meadowns, D. L.; Randers, J. y Beheren, W. W. (1972): Limits to growth, Potomac Associates, New York.

Meadows, D.; Randers, J. y Meadows, D. (2004): Limits to growth: the 30 year update, Chelsea Green Publishing.

6 Samuelson, P. (1947): Foundations of Economic Analysis, Cambridge, Mass., Harvard University Press.

7 Op. cit.

8 Pareto, V. (1897): Cours d'Economie Polique, Lausanne.

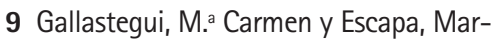
ta (2007): "El Medio Ambiente y las Empresas ¿Oportunidad o Restricción?", Papeles de Economía Española, n. 112.

10 Op. cit.

11 Daly, H. E. (1974): "The economics of the steady state", American Economic Review, 64(2).

12 Un tanque de petróleo se consumirá de forma sostenible sólo si parte de los beneficios obtenidos con su uso se invierten sistemáticamente en parques eólicos, placas fotovoltaicas, plantaciones de árboles, de forma tal que cuando el petróleo se haya agotado todavía esté disponible un flujo equivalente de energía renovable.

Recibido: 30 de junio de 2010

Aceptado: 31 de octubre de 2010

13 Wakerganel, M. y Rees, W. (1996): Our Ecologial Footprint: Reducing the
Human Impact on the Earth, New Society Publishers, Gabriola Island, British Columbia.

14 Stern, N. (2008): "The Economics of Climate Change", American Economic Review, 98 (2).

15 Gallastegui, M. Carmen y Chamorro, J. M. (2008): Medio Ambiente y la Necesidad de Acuerdos Internacionales. Tratado de Regulación del Sector Eléctrico, vol. 2, Ed. Thompson Aranzadi.

\section{BIBLIOGRAFÍA}

Baumol, W. J. y Oates, W. E. (1988): The Theory of Environmental Policy, Externalities, Public Outlay and the Quality of Life, New Jersey, Printice Hall, Englewood Cliffs.

Daly, H. E. (1974): "The economics of the steady state", en American Economic Review, 64(2).

Malthus, T. R. (2009): Ensayo sobre el principio de la población, Madrid: Minerva.

Jevons, W. S. (1865): The coal question: An inquiry Concerning the Progress of the Nation and the Probable Exhaustion of our Coal Mines, Flux, W. (ed).

Meadows, D. H.; Meadowns, D. L.; Randers, J.y Beheren, W. W. (1972): Limits to growth, New York: Pontomac Associates.

Meadows, D. y Randers, J. (2004): Limits to growth: the 30 year update, Chelsea Green Publishing.

Gallastegui, M. ${ }^{a}$ Carmen y Chamorro, J. M. (2008): Medio Ambiente y la Necesidad de Acuerdos Internacionales. Tratado de Regulación del Sector Eléctrico, vol. 2, Ed. Thompson Aranzadi.

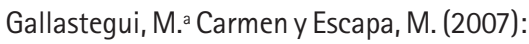
"El Medio Ambiente y las Empresas ¿Oportunidad o Restricción?", Papeles de Economía Española, n. ${ }^{\circ} 112$.
Pareto, V. (1897): Cours d'Economie Polique, Lausanne. 
Pigou, A. (1920): The Economics of Welfare, London: MacMillan.

Samuelson, P. (1947): Foundations of Economic Analysis, Cambridge: Mass., Harvard University Press.
Stern, N. (2008): "The Economics of Climate Change", en American Economic Review, n. 98.

Raupach et al. (2007): Global and regional drivers of accelerating $\mathrm{CO}_{2}$ emissions, PNAS.
Wakerganel, M. y Rees, W. (1996): Our Ecologial Footprint: Reducing the Human Impact on the Earth, Gabriola Island: New Society Publishers, British Columbia. 